\title{
Homologous Recombination under the Single-Molecule Fluorescence Microscope
}

\author{
Dalton R. Gibbs 1 and Soma Dhakal * (1) \\ Department of Chemistry, Virginia Commonwealth University, 1001 West Main Street, \\ Richmond, VA 23284, USA; gibbsdr@vcu.edu \\ * Correspondence: sndhakal@vcu.edu
}

Received: 29 October 2019; Accepted: 30 November 2019; Published: 3 December 2019

check for updates

\begin{abstract}
Homologous recombination (HR) is a complex biological process and is central to meiosis and for repair of DNA double-strand breaks. Although the HR process has been the subject of intensive study for more than three decades, the complex protein-protein and protein-DNA interactions during HR present a significant challenge for determining the molecular mechanism(s) of the process. This knowledge gap is largely because of the dynamic interactions between HR proteins and DNA which is difficult to capture by routine biochemical or structural biology methods. In recent years, single-molecule fluorescence microscopy has been a popular method in the field of HR to visualize these complex and dynamic interactions at high spatiotemporal resolution, revealing mechanistic insights of the process. In this review, we describe recent efforts that employ single-molecule fluorescence microscopy to investigate protein-protein and protein-DNA interactions operating on three key DNA-substrates: single-stranded DNA (ssDNA), double-stranded DNA (dsDNA), and four-way DNA called Holliday junction (HJ). We also outline the technological advances and several key insights revealed by these studies in terms of protein assembly on these DNA substrates and highlight the foreseeable promise of single-molecule fluorescence microscopy in advancing our understanding of homologous recombination.
\end{abstract}

Keywords: single-molecule fluorescence microscopy; fluorescence resonance energy transfer (FRET); homologous recombination (HR); DNA break repair; DNA curtain; optical tweezers; Holliday junction (HJ)

\section{Introduction}

Homologous recombination (HR) is a well-conserved and essential biological process for both genetic exchange and the repair of double-stranded DNA (dsDNA) breaks [1-4]. The HR process has been a hot research topic for decades, initially due to the quest for unraveling the fundamental mechanisms of HR, its role in DNA replication, and in rescuing stalled replication forks [1,5]. Later HR's role in DNA damage repair rose to significance, due to the fact that double-stranded DNA breaks are a form of DNA lesion that, if left unrepaired, can lead to genetic instability, genetic defects, and certain cancers which, as a consequence, is highly dangerous to cells $[1,6,7]$. The details of the HR process including DNA mismatch repair have been previously discussed $[1,8,9]$ and reviews covering the entire single-molecule perspective on HR, including studies using force microcopy and tethered particle motion, have been published $[7,10,11]$. However, the focus of these previous reviews/surveys has been from a biological perspective, detailing knowledge of the HR pathway. The single-molecule fluorescence microscopy studies of HR have been quite extensive, but these studies thus far are limited to only a few labs across the world. Therefore, in this review, we intended to bring this area of study to focus as an effort to allow current and new researchers to realize the capabilities and opportunities of single-molecule fluorescence microscopy techniques and potentially help adopt these techniques to further our mechanistic understanding of HR. 
Repairing dsDNA breaks involves three phases-pre-synaptic, synaptic, and post-synaptic - corresponding to the trimming of the dsDNA backbone creating single-stranded DNA (ssDNA) overhangs, formation of a homologous exchange intermediate called a Holliday junction $(\mathrm{HJ})$, and, finally, the resolution of the junction back into dsDNA [1,7]. A number of enzymes are responsible for performing one or more roles in these processes for successful execution of DNA break repair. However, due to the involvement of the same enzymes in more than one specific function, there is still a limited understanding of their roles in the HR process.

The groundwork towards unraveling complex pathways of the HR process have been laid out initially through a number of biochemical studies. The protein-DNA complexes that form during the HR process have been revisited more recently via single-molecule approaches and have significantly improved our understanding of the process [19-25]. For example, the bulk biochemical work by West's group $[24,26,27]$ and Ogawa's group $[28,29]$ provided a better understanding of structure-function relationships of several HR enzymes. However, due to the inherent averaging of these bulk assays and inability to reveal dynamic aspects of molecular interactions, these studies provided only limited information. Monitoring of real-time biomolecular interactions typically requires a more direct approach such as high-resolution microscopy. Recent advancement in fluorescence microscopy approaches presents a powerful tool to enable direct visualization of protein-protein and protein-DNA interactions that are critical to HR. In fact, because these techniques bypass ensemble averaging, they allow real-time visualization of the activity of individual fluorophore-labeled molecules, ultimately providing unprecedented insights into the subcellular world that would otherwise not be possible to access. For example, a fluorescence resonance energy transfer (FRET) based approach provides a signal based on the distance between the donor and acceptor fluorophore (Figure 1A) and, thus, it has been used to study HR proteins acting on DNA [30,31]. Using simple fluorescence-based imaging, both single- and double-stranded DNA curtains have been developed to study filament-forming proteins (Figure 1B). By using a fluorescently labeled HJ structure, the binding of $\mathrm{HJ}$ resolvases have been probed (Figure 1C).
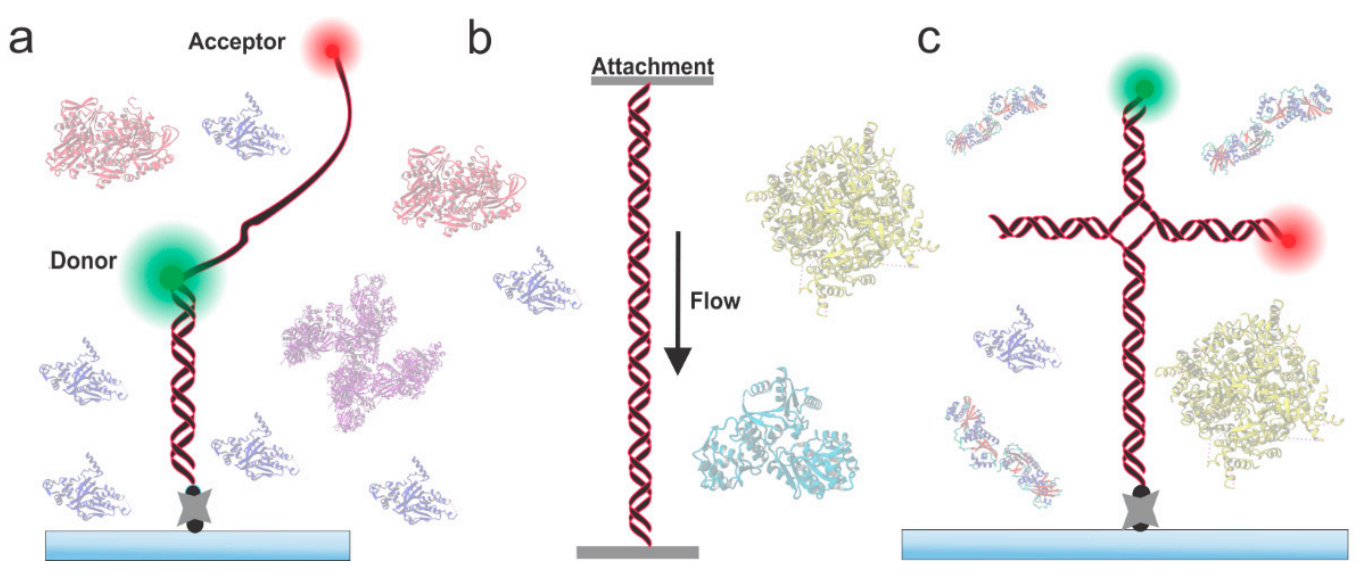

Figure 1. Three key DNA substrates (i.e., single-stranded DNA (ssDNA), double -stranded (dsDNA), and Holliday Junction (HJ)) of the homologous recombination (HR) process. (a) Typical fluorescence resonance energy transfer (FRET) based DNA construct designed to study ssDNA-binding proteins. These constructs generally consist of a dsDNA region affixed to the surface of a microscope slide and a flanking ssDNA region for protein binding. The constructs are functionalized with a pair of fluorophores (donor and acceptor) to enable FRET-based imaging and to monitor protein binding. (b) Generalized representation of dsDNA curtain, wherein a dsDNA is stretched between two micro-fabricated attachment points. Fluorescently labeled proteins can then be used to study binding. (c) Surface-bound $\mathrm{HJ}$ analog used in FRET-based imaging. Structures on the background are the crystal structures of some of the HR proteins [12-18]. 
Various protein systems that are involved in the repair of dsDNA breaks via the HR pathway have several members, some of which act on both the dsDNA and ssDNA substrates. For instance, RecBCD is a complex of three enzymes which cuts and separates an ssDNA from genomic dsDNA at the chi sequence, leaving in its wake a trail of RecA protein bound to the ssDNA. The creation, protection, and repairing of the ssDNA segments is essential in the dsDNA break repair pathway. Typically, the ssDNA segments are protected by single-stranded DNA binding proteins (SSBs), where the SSBs wrap around the ssDNA portions to protect them from other cellular processes [32] and facilitate their roles in HR. For example, although RecA is mostly known for its ssDNA-binding activity, it plays a key role in synapsis by binding to homologous dsDNA [33]. The RecA homolog, Rad51, serves a similar role in humans as a key player in the formation of synapsis with both ssDNA and dsDNA binding activity. Another key protein is RecQ, which is a helicase with various roles such as unwinding of dsDNA and promoting DNA annealing [34]. Similarly, the replication protein A (RPA) is an indispensable player in HR among other important DNA metabolic pathways in eukaryotes [2]. Further, the repair of single-strand gaps in dsDNA is accomplished by the enzyme complex RecFOR using a homologous mechanism [35-37]. On the other hand, the resolution of the HJ cross-strand intermediate formed among the homologous DNA molecules during the late-stage of $\mathrm{HR}$ is recognized and resolved by the protein complex RuvABC (composed of three proteins RuvA, RuvB, and RuvC) in prokaryotes [27] and different sets of proteins in eukaryotes. In eukaryotes, the RuvABC protein complex builds upon the $\mathrm{HJ}$ in a sequential manner with RuvA locating and binding to the HJ, RuvB binding to the RuvA-HJ complex and initiating branch migration, and RuvC later binding to and resolving the $\mathrm{HJ}$ [38]. As we work through this review, some of the proteins will appear in multiple sections, as they have both ssDNA- and dsDNA-binding activities. In this review, we discuss these recent advances in the HR field that have harnessed the unique ability of single-molecule fluorescence microscopy to reveal unprecedented molecular-level details of critical protein-DNA complexes.

\section{Probing HR Proteins Operating on ssDNA}

The ssDNA-binding HR proteins have largely been explored using single-molecule FRET (smFRET) or DNA curtain platforms. The DNA constructs used in smFRET studies generally follow a simple design as shown in Figure 1A, wherein a region of dsDNA is bound to the surface of the microscope slide with a short biotin-modified ssDNA overhang [39-42]. The construct is generally labeled with a single fluorophore to enable fluorescence imaging or a donor-acceptor pair to enable FRET (Figure 1). The FRET-labeled constructs are particularly suitable for studying filament-forming HR proteins (e.g., Rad51 and RPA) that bind to the exposed single-stranded region and modify its length, shape, and/or stiffness [39-42]. The FRET techniques, being sensitive to sub-nanometer distance changes between the donor and acceptor fluorophores [30,31], allow any change in the ssDNA conformation or length to be directly translated to FRET. This powerful yet simple technique can provide unique insights into the nature of binding and unbinding of proteins. The DNA curtain techniques, on the other hand, use linearized pieces of DNA anchored on a fixed platform on one or both ends while fluorescently-labeled species of interest, such as HR proteins, assemble upon them [43]. These techniques can utilize ssDNA or dsDNA molecules, and both of them will be discussed in this review. The assembly and activity of these differently labeled proteins of interest can be studied using fluorescent colocalization, a technique where the emissions of two or more species are tracked in space, and the relative localization and motion of these species are used to infer interactions and/or function. Unlike smFRET, where at least two different types of fluorophores (acceptor and donor) are used and the ratio of emissions of the donor and acceptor are monitored to determine the FRET efficiency (and ultimately the donor-acceptor distance), emissions in space are used to determine the locations of molecules in the colocalization experiments. Some of the key advantages of the DNA curtain platform are the ability to carry out highly parallelized localization experiments of DNA binding/processing protein(s) on single extended DNA molecules using proteins tagged with 
fluorophores and/or DNA-specific fluorescent dyes [43]. Below, we discuss recent single-molecule studies of HR proteins operating on ssDNA.

Single-strand DNA binding proteins (SSBs) and their analogs are essential in protecting ssDNA regions in various biological processes such as preventing the formation of ssDNA secondary structures and assisting in recruiting certain RecA mediators [32]. The displacement of SSBs from ssDNA substrate is one of the first steps in homologous recombination. This is generally accomplished by RecA displacing the protein through formation of its own filaments around the ssDNA [32]. Bell et al. [44], in 2015, studied the assembly of fluorescently labeled SSBs onto ssDNA using a DNA curtain technique where ssDNA molecules that were tethered at one end were extended by a gentle buffer flow and visualized using fluorescence microscopy. This study determined that the ssDNA is wrapped around SSB tetramers in 30-70 nucleotide sections, and that SSB exchanges with the solution protein regularly [44]. A study by Joo et al. [39], in 2006, further clarified the reported interaction between SSB and RecA, where the filament-forming ability of RecA on SSB-saturated DNA was measured using a FRET system. This study showed that RecA displaces SSB at normal filament formation rates as long as this displacement travels outward from a pre-formed RecA nucleation site, demonstrating that no active mechanism to clear SSB off ssDNA is required [39]. The direct binding of human SSB analog SSB1 in complex with RPA to ssDNA was demonstrated using single-molecule FRET in 2013 by Yang et al [41]. This study found that the SSB1 complex binds to ssDNA with a lower affinity in the absence of RPA [41]. Bell et al. [37] further explored the interaction between SSB and RecA, in 2012, using a variant of the DNA curtain method, where surface-tethered SSB-bound ssDNA molecules were extended linearly using a gentle flow and then exposed to fluorescently labeled RecA. The rate and nature of RecA filament formation in SSB-bound conditions was then monitored. This study demonstrated that the RecA nucleation starts at various points along the strand with the binding of a dimer and radiates out from these points of nucleation in either direction with some preference for $5^{\prime}$ to $3^{\prime}$ filament growth. This study also showed that the RecA nucleation is facilitated by RecFOR, and the speed of filament growth is enhanced by RecOR [37].

The properties and functions of RecA filaments have been the subject of major interest for single-molecule fluorescence studies in various stages of the HR process [45]. In 2012, Ragunathan et al. [42] studied RecA's role in homology search using a FRET system with an acceptor fluorophore integrated into the ssDNA portion of the DNA construct and a donor fluorophore integrated on the dsDNA. They found that the dsDNA "slid" across the RecA filament at a speed that allowed more DNA to be sampled in homology search than previously suspected [42]. Kim et al. [46] studied the effect of sequence on the formation of the RecA filament using a FRET construct with fluorophores attached among sequences of interest. This group found that the chi sequence (5'-GCTGGTGG) does not itself promote nucleation, but that decreased monomer exchange due to the RecA's affinity for TGG repeats increases the amount of time that RecA pauses at this sequence, promoting nucleation [46]. Kim et al. [40] showed that the RecA filament switches between a relaxed and stretched conformation in the presence of adenosine di-phosphate (ADP) and adenosine tri-phosphate (ATP), respectively (Figure 2A). This switching between relaxed and stretched conformations allows the filament to remain assembled during various stages of HR. This is due to the model of the filament stretching and relaxing cycle, in which cooperative structural changes within adjacent RecA monomers allow the filament to stay in its current state in the midst of ATP exchange [40]. 
a


Figure 2. Typical DNA constructs to study ssDNA-binding proteins. (a) The formation of Rad51 filament around the ssDNA changes FRET efficiency due to ATP-dependent extension of the ssDNA (middle panel). The bottom panel shows a typical single-molecule FRET-time trace illustrating the delay between the buffer exchange and conformational switch from the stretched to compressed states. This figure is reprinted with permission from Kim et al. [40] and any further inquiries for permissions should go to ACS. (b) Depicted is a procedure for making ssDNA curtains and a schematic of the rolling circle replication (RCR) to produce ssDNA molecules for ssDNA curtains using a biotinylated primer, M13mp18 circular DNA, and $Ф 29$ DNA polymerase ( $\$ 29$ DNAP). The ssDNA molecules are anchored to a surface coated with a lipid bilayer; flow is then used to extend the DNA which then anchors to downstream barriers non-specifically. Green fluorescent protein (GFP)-labeled RPA then assembles in a filament around the ssDNA, which can be visualized using fluorescence microscopy. In this specific example, a mixture of Rad51 and ATP was incubated with the curtain and Rad51-mediated displacement of GFP-RPA was monitored. Adapted with permission from Elsevier [43]. 
The Rad51 nucleation was studied using an ssDNA construct with a FRET pair on the single-stranded region by Lu et al. [47]. This study found that accessory factors, Swi5-Sfr1, stabilize the Rad51 monomers allowing for an enhanced filament formation [47]. In 2012, Gibb et al. [43,48] established a technique for visualizing DNA-binding proteins on an ssDNA curtain. In this method, ssDNA molecules created through a process called rolling circle replication (RCR) are attached to biotinylated lipids that form a part of a lipid bilayer coating on a slide surface. The ssDNA molecules are lined up on a nanofabricated ridge and then extended using a buffer flow (Figure 2B) [43,48]. Gibb et al. [49] used this platform in 2014 to anchor dsDNA curtains to study the presynaptic interplay of yeast RPA, Rad51, and Rad52. Using fluorescently labeled proteins, they determined that Rad52 binds to RPA and prevents RPA unbinding. This result implicated that a RPA-Rad52-Rad51 intermediate has a significant role in late-stage HR [49]. In 2017, Ma et al. [50] used this approach to study the assembly and disassembly of Rad51 filaments. In this study, the rate of displacement of a fluorescently labeled RPA was used to determine that the presence of RPA in solution inhibits Rad51 filament formation but not extension. This result suggests that RPA has a role in Rad51 regulation and potentially modulates the properties of already assembled Rad51 filaments [50]. Myler et al. [51] used an ssDNA curtain technique and fluorescently labeled proteins to determine the effect of RPA on ExoI binding. Using fluorescent colocalization microscopy, they showed that ExoI binding was interrupted by the binding of RPA and that SSB1 enhanced the binding of ExoI. This result indicates that SSB1 and RPA have a significant role in regulating the resection of DNA by Exo1 in DNA damage repair in humans [51].

In addition, the ssDNA platform has also been used to study other HR proteins. For example, in 2017, De Tullio et al. [52] used ssDNA curtains to study the assembly of Srs2 onto Rad51-bound sDNA. This study found that Srs2 moves along the DNA unidirectionally, displacing Rad51 in an ATP-dependent manner. When Rad52 was introduced and allowed to bind to the Rad51 filament, it had no effect on the ability of Srs 2 to displace the Rad51 from ssDNA [52]. Later, the same group found that Srs2 displaces Rad51 in a unidirectional manner, and that Srs2 begins this displacement from points where RPA has already bound, clearing the way for more RPA molecules which then recruit more Srs2 molecules [53]. The binding of Rad52 to both ssDNA and dsDNA was explored using an optical tweezers system and fluorescently labeled Rad52 in 2017 by Brouwer et al [54]. While Rad52 was found to have a strong and static binding with ssDNA, it binds to dsDNA less tightly in a transient manner. Using force spectroscopy, they were also able to determine that Rad52 helps stabilize dsDNA to prevent melting, which could be advantageous during certain steps of recombination [54]. In 2019, Crickard et al. [55] explored the relationship between highly similar proteins RPA and Dmc1 both of which are present in cells during meiotic HR and have very similar binding behaviors. Using fluorescently labeled proteins, they determined that, while both proteins form filaments under similar conditions, Dmc1 binds specifically to the Hop2-Mnd1 complex that has been implicated in recruiting dsDNA to the synaptic complex during meiotic HR [55].

\section{Probing HR Proteins Operating on dsDNA}

Investigation of dsDNA-binding HR proteins has been an area of considerable work in the fluorescence microscopy field. These proteins are critical to the presynaptic and synaptic stages of HR and often work in concert. Therefore, bulk methods have difficulty analyzing these complex interactions. Studies investigating and visualizing the binding of these HR proteins are by large performed on some linearized piece of dsDNA molecules hold in place under a fluorescence microscope. The mechanism by which the dsDNA molecules are held in place can be a "DNA curtain" approach, where the dsDNA molecules are bound to a functionalized slide surface on one end with continuous buffer flow to hold the DNA linear or are bound on both ends. Another method for holding a dsDNA molecule linear is binding the DNA to a functionalized polystyrene bead which is then held in a moveable optical trap, using a flow to keep the DNA extended. Exposure of DNA to different buffers 
is facilitated by physically moving the bead into various areas of a flow cell with buffers separated by laminar flow.

The RecA filament formation was studied in 2006 by Galletto et al. [45] using dsDNA held in a movable optical trap. This dsDNA was exposed to fluorescently labeled RecA in various concentrations, and the binding was tracked using a fluorescence microscope. This strategy allowed them to determine that the length of dsDNA increased by $\sim 70 \%$ upon RecA filament formation (Figure 3). They also showed that the RecA nucleation was dependent on the presence of a nucleoside triphosphate but the filament growth after nucleation could occur bidirectionally around the nucleation site with or without the cofactor [45]. In 2012, Forget et al. [56] used an optical trap system to study the physical nature of RecA's role in homology search, a key step in HR. In this study, they used fluorescently labeled RecA and, by monitoring the end-to-end distance of the dsDNA molecule, they demonstrated that the rate of homology search varies with the degree of linearization of the DNA molecule. They attributed this observation to a 3D homology search mechanism where the RecA protein samples 3D space around it rather than linearly down a single DNA molecule, a mechanism they called intersegmental contact sampling. According to this model, the RecA filament has a polyvalent surface which can sample several non-contiguous parts of adjacent dsDNA; this in combination with the globular nature of dsDNA under relaxed conditions allows the RecA filament to sample numerous nearby neighboring sequences during its search for homology [56].

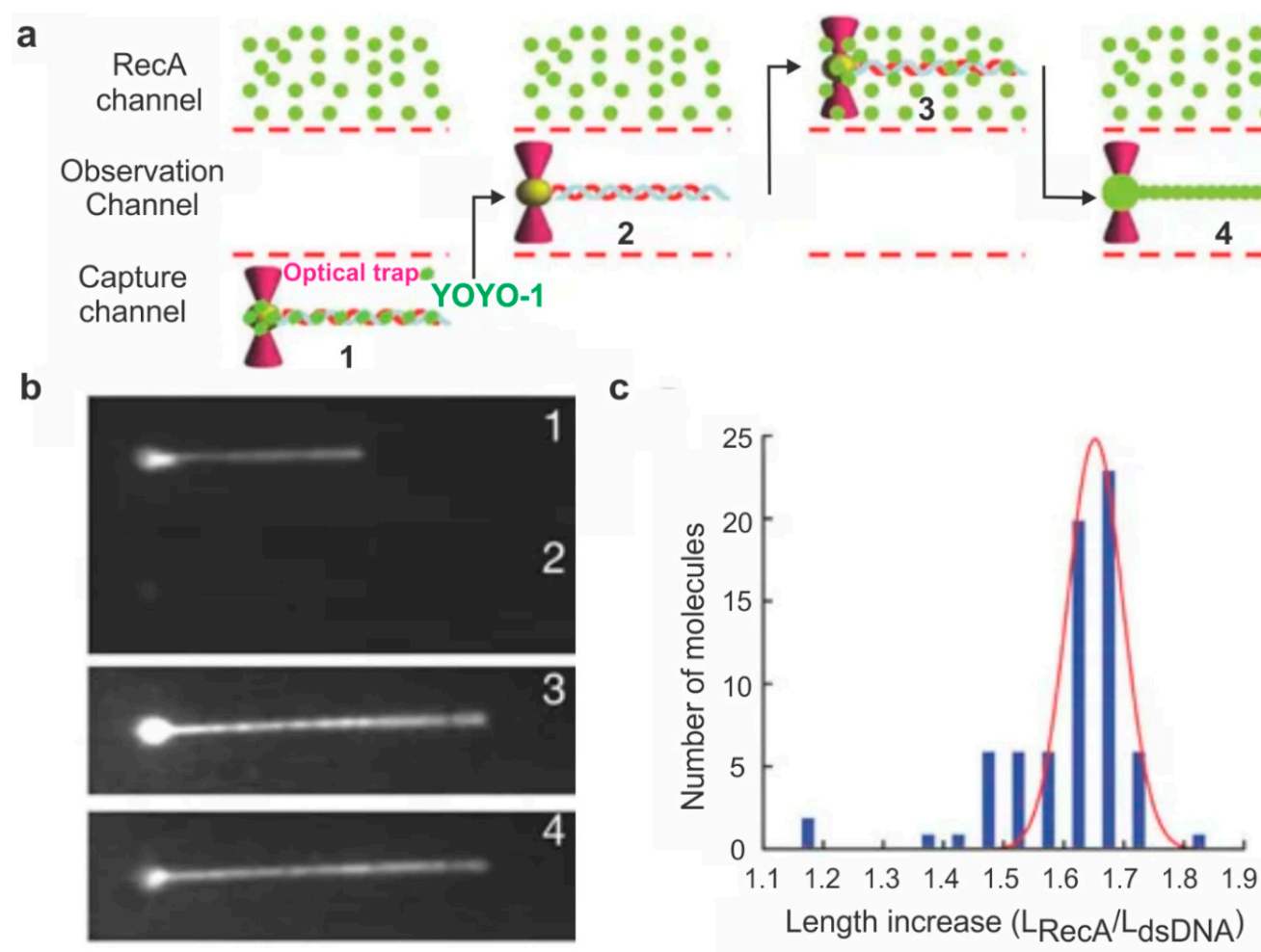

Figure 3. Optical tweezers-based monitoring of RecA assembly on dsDNA. (a) Experimental setup used to study the effect of RecA on dsDNA length. Biotin-functionalized lambda DNA stained with a DNA-binding dye called YOYO-1 was attached to a bead held in a moveable optical trap. This DNA was held linear with a constant laminar flow, and the bead was moved to various channels (shown by arrows) either to expose the DNA to protein or to visualize it. (b) The DNA was first located, and its length was determined. The bead was then moved to the observation channel where YOYO-1 dissociates from the DNA and then to a channel containing fluorescently labeled RecA. The length of the DNA was again recorded after RecA binding. Images of the positions of the DNA bead complex as described in (a). (c) Histogram showing the distribution of the length change of DNA with $\left(L_{\text {RecA }}\right)$ and without $\left(L_{\mathrm{dsDNA}}\right)$ RecA. This figure is reprinted with permission from Springer Nature [45], copyright Springer Nature 2006. 
In 2001, Bianco et al. [57] published a study visualizing the DNA unwinding activity of the RecBCD enzyme complex. In this study, they bound an end-functionalized dsDNA molecule to a surface-functionalized polystyrene bead and held the bead in an optical trap. The DNA was then exposed to a DNA-binding fluorescent dye called YOYO-1 to visualize the DNA. This fluorescent DNA was exposed to the RecBCD complex which processed through the DNA releasing the florescent dye thus allowing the direct measurement of the rate of RecBCD procession along the DNA. This study demonstrated that the unwinding of RecBCD proceeds continually at $\sim 0.3 \mu \mathrm{m} \mathrm{s}^{-1}$ until this protein complex dissociates [57]. This work was also complemented by the crystal structure of RecBCD obtained by Singleton et al. [58]. In 2012, Yang et al. [59] studied RecBCD's affinity for the chi sequence more closely using the same technique as Bianco et al. [57]. In this study, they showed that the specificity of RecBCD towards the chi sequence was either compromised or completely abolished when a suspected chi-recognition domain was mutated [59]. In 2013, Liu et al. [60] used optical tweezers to study the variation in rates of RecBCD's unwinding kinetics. In this study, processing of DNA by RecBCD was interrupted by moving the optical trap to a buffer channel containing no ATP and then moved back to the ATP-containing channel to reinitiate the RecBCD activity. Using this approach, they observed that the DNA unwinding rate of individual enzyme molecules varies. They attributed this observation to the ATP-dependent stabilization of one of many equilibrium sub-states, each of which has a slightly different conformation and, thus, exhibits a different progression rate [60].

The essential HR protein, Rad51's, filament-forming behavior was studied in 2006 by Prasad et al. [61] using a DNA curtain platform wherein the length of YOYO-1 labeled dsDNA was monitored before and after binding of Rad51. As Rad51 bound to the dsDNA, the overall length of the DNA molecules increased due to the extended conformation of DNA upon formation of the Rad51 filament. Further, after systematic mutation of Rad51 in various domains, it was found that the protein's L1 domain was essential for filament formation [61]. In the same year, the Greene [62] lab developed a new platform for studying protein-DNA interactions, called DNA curtain, in which several dsDNA molecules are stretched and attached on both ends creating a parallel array of DNA molecules. The parallel array of DNA molecules can be visualized via total internal reflection fluorescence (TIRF)-based single-molecule fluorescence microscopy by directly staining the DNA molecules with fluorescent dyes. On the application side, protein-DNA interactions can be studied on the DNA curtain platform using fluorescently tagged proteins. For example, Yeykal et al. [62] used this method to show that a GFP-tagged Rad51 can diffuse along the dsDNA molecules in a one-dimensional random walk using only Brownian forces.

The double-bound ssDNA curtain (Figure 4) provided an exceptional platform for investigating the homology search function of Rad51. In 2015, Qi et al. [63] used this method to study the progression of fluorescently tagged homologous dsDNA along a Rad51 filament built up around the curtain. They were able to determine that Rad51 uses a length-based mechanism that looks in segments of 8 nucleotides to speed up the search process [63]. In another application, Rad et al. [64] used a dsDNA curtain system to visualize the helicase unwinding activity of RecQ. In this study, they showed that, as RecQ unwinds the dsDNA, the fluorescently labeled SSB binds. This system visualized not only the rate and number of ssDNA regions created upon RecQ exposure but also that the ssDNA forks were formed upon exposure. More interestingly, they were able to determine that RecQ dimers are required to initiate this unwinding process and that unwinding proceeds at a rate of $40-60 \mathrm{bp} \mathrm{s}^{-1}$ [64]. 
a

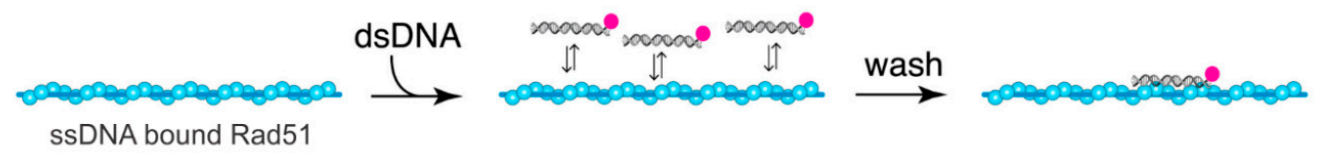

b

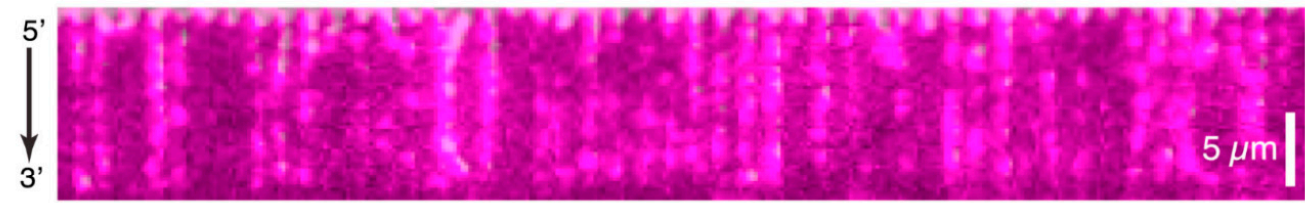

C
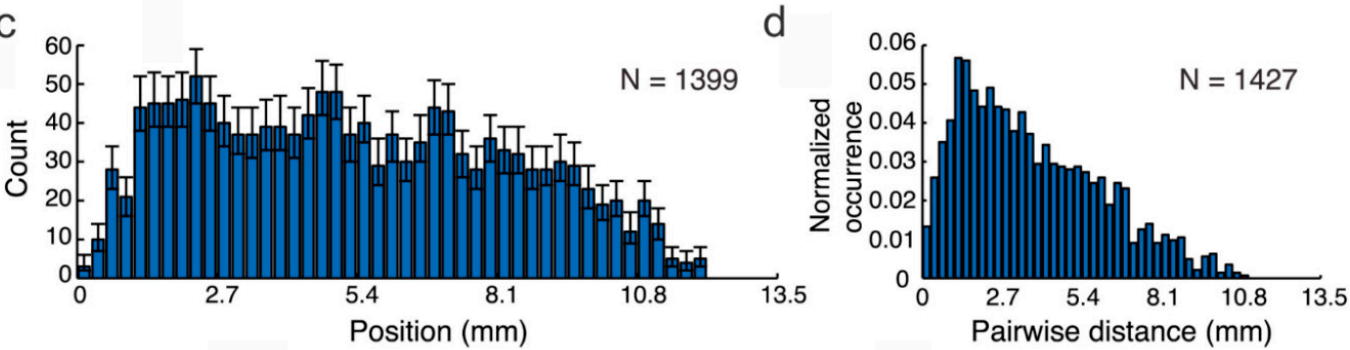

Figure 4. The ssDNA curtain used to study the progression of dsDNA fragments along a Rad51 filament. (a) The strategy for visualizing dsDNA binding to the Rad51-modified ssDNA. The dsDNA was fluorescently labeled. (b) The DNA curtain displaying the binding of dsDNA on Rad51-modified ssDNA. (c) The binding site distribution on the DNA measured from the $5^{\prime}$-end of the DNA and (d) the distance between fluorophores on the Rad51 filament. This figure was reprinted with permission from Elsevier [63], copyright 2015.

\section{Probing HR Proteins Operating on Holliday Junctions}

Studying proteins that bind to the $\mathrm{HJ}$ using single-molecule microscopy presents interesting challenges and opportunities due to the inherent conformational changes that occur in the HJ $[27,65,66]$. The HJ is a naturally dynamic structure that switches between two stacked conformers many times per second through an open cruciform intermediate [65]. In recent years, the dynamic nature of the junction has been exploited to probe the interaction between the junction and HR proteins. This is generally done by attaching a pair of fluorophores on the ends of adjacent arms of the HJ to enable FRET. Due to the dynamic nature of the HJ, it results in a distinct FRET efficiency for each conformer.

Single-molecule studies of the HJ have determined the structural dynamics of the HJ, particularly the shape that the junction adopts and the circumstances (primarily ionic environment and nucleotide composition at the core of the junction) under which it would switch from one stacked conformer to another. In 2003, McKiney et al. [65] used an HJ labeled with a FRET pair on adjacent arms and a $\mathrm{Ca}^{2+}$-rich buffer to determine the energy landscape which governs the switching of the HJ between its stacked conformers. They concluded that the conformational switch must occur during many intermediate stages of HR [65]. The stepwise random progression of branch migration (the migration of the four-way junction along the DNA axis) was experimentally visualized by Karymov et al. [67] in 2005. This study showed that the junction switches between its stacked and cruciform conformations; the cruciform being more favorable to branch migration, as it allows the $\mathrm{HJ}$ to migrate several base pairs at once. They also found that, as the concentration of $\mathrm{Mg}^{2+}$ increased, the $\mathrm{HJ}$ switched to its stacked conformation more often, stalling the branch migration [67]. The relative abundance of the HJ cruciform intermediate in solution was determined by Joo et al. [68]. They also proposed that this cruciform conformation could potentially be stabilized through protein binding [68]. The conformational switching of the HJ was further studied by Hohng et al. [69] using a surface-bound and bead-tethered HJ labeled with a FRET pair. This system allowed the researchers to apply force to the HJ and monitor its conformation via FRET signal. In this manner, they were able to map two-dimensional energy landscapes of conformational switching of the $\mathrm{HJ}$ [69]. The binding interaction of the MutS $\gamma$ analog 
Msh4-Msh5 to HJs and other types of DNA structures was probed in depth by Lahiri et al. [70,71] in 2018, discovering that the MutS $\gamma$ stabilizes the $\mathrm{HJ}$ and prevents branch migration until resolution by MutL $\gamma[70,71]$.

Overall, the direct visualization of HJ-binding proteins is an ongoing research effort with only a few HR proteins having been studied. In 2015, Iwasa et al. [72] investigated the assembly of RuvB onto a RuvA-bound HJ using fluorescently tagged RuvB and HJ (Figure 5A). In this study, through the counting of fluorescence photobleaching events, they were able to determine that six RuvB molecules assemble around the DNA arm of the HJ. They also determined that the RuvB's assembly around the RuvA-bound HJ was an ATP-dependent process [72]. The geometry of the RuvA-bound HJ was studied using a FRET-labeled HJ construct in 2018 by us [66]. In this study, we demonstrated that, upon binding to the junction, RuvA ceases the dynamics of the HJ. The observed FRET efficiency of the RuvA-HJ complex indicated that the RuvA traps the $\mathrm{HJ}$ in its open cruciform conformation. Additionally, experiments at various concentrations of $\mathrm{Mg}^{2+}$ and $\mathrm{Na}^{+}$showed a strong relationship between the ion concentration and RuvA binding activity (Figure 5B), with a compromised binding at higher concentration of $\mathrm{Mg}^{2+}$, suggesting that the RuvA-HJ interaction is primarily electrostatic in nature [66].

In 2019, Zhou et al. [73] used FRET-labeled HJs to study the binding of late-stage HJ resolvases. In this study, the group used a high ionic strength buffer composed of calcium chloride to slow down the conformational switching of the HJ without interfering with the RuvC binding. Using this system, they studied the binding of T7 endonuclease 1, RuvC, GEN1, and hMus81-Eme1 to the HJ and found that they all shared the same dynamic HJ activity while bound. They concluded that these enzymes, all dimers, use multivalent binding to allow the $\mathrm{HJ}$ to freely engage in conformational switching (Figure 6), revealing several interesting mechanistic roles of these enzymes which had thus far not been explored [73]. 
a
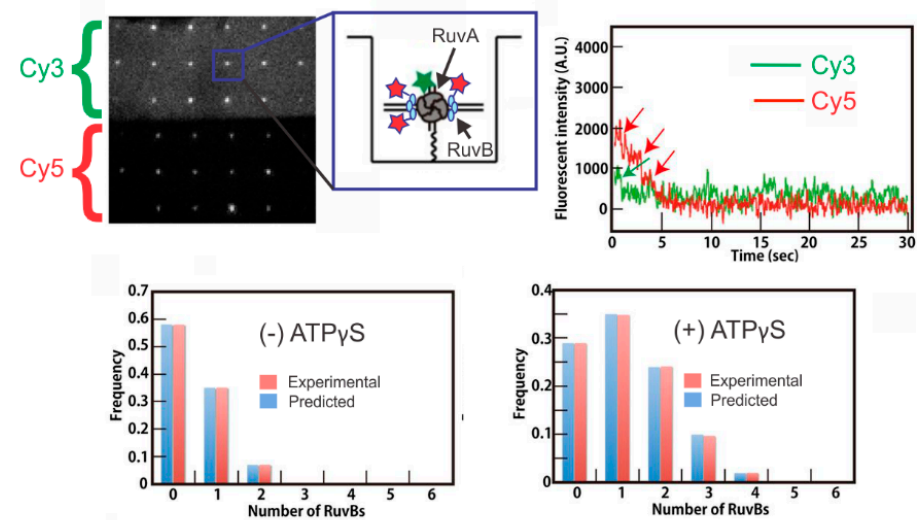

b
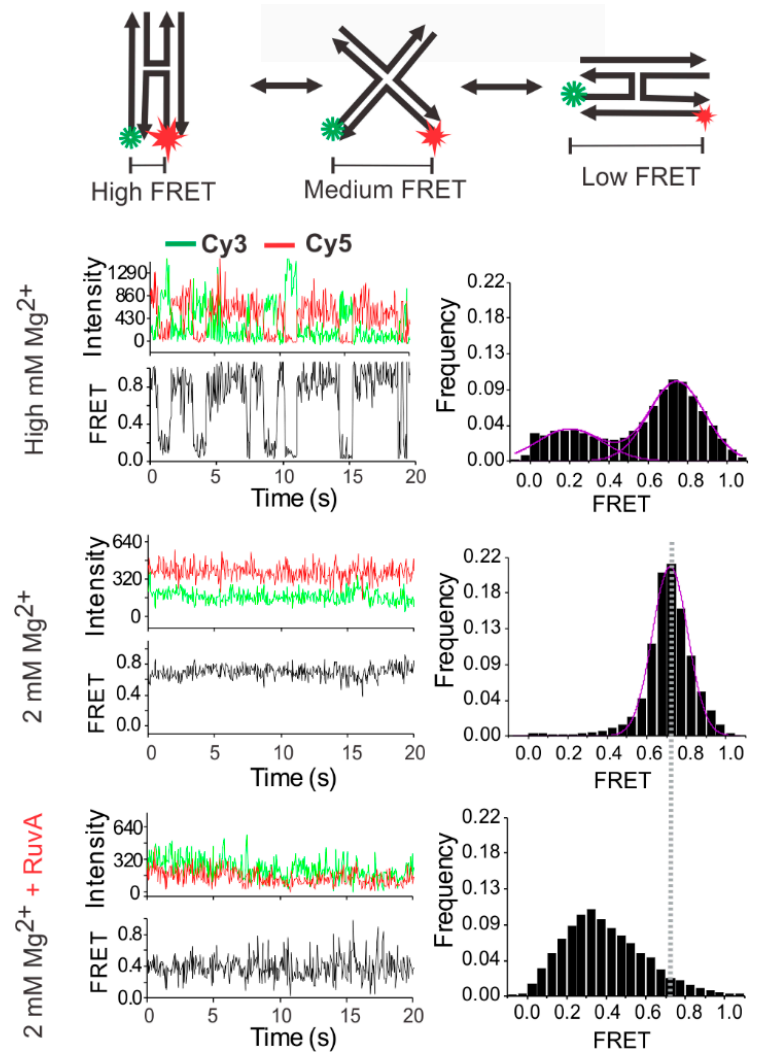

Figure 5. Probing the binding interaction between HR proteins and HJ. (a) Single-molecule waveguide experiment on a RuvAB-HJ complex. $\mathrm{Cy} 3$ and $\mathrm{Cy} 5$ channels represent the fluorescence emission from these respective fluorophores. Inset depicts a RuvAB-HJ complex, where RuvA is labeled with a Cy3 fluorophore (green), and RuvB is labeled with a Cy5 fluorophore (red). The top right panel depicts the photobleaching events for counting the number of RuvA and RuvB molecules in the complex. The bar graphs demonstrate the number of RuvBs determined based on the photobleaching events in the absence and presence of ATP $\gamma$ S. This figure was adapted with permission from Iwasa et al. [72]. (b) Probing the binding of RuvA to the junction using single-molecule TIRF microscopy. Top panel: Schematic of HJ dynamics between two isomers via an open-state conformation. At high $\mathrm{Mg}^{2+}$ concentration without RuvA, the switching between the two isomers was clearly captured (typically $>50 \mathrm{mM} \mathrm{Mg}^{2+}$ is known to slow down the switching). The occupancy of the low ( $\sim 0.2$ FRET) and high ( $\sim .8$ FRET) FRET efficiency states were at $\sim 70: 30$ distribution. Decreasing the $\mathrm{Mg}^{2+}$ concentration to $2 \mathrm{mM}$, the rapid switching between the two stacked conformations resulted in weighted average of the FRET efficiency of $\sim 0.7$. Interestingly, when the HJ was exposed to RuvA, the FRET efficiency shifted to $\sim 0.38$, demonstrating that RuvA clamps the $\mathrm{HJ}$ in its open conformation. This figure was adapted with permission from the American Chemical Society [66], copyright (2018) American Chemical Society. 


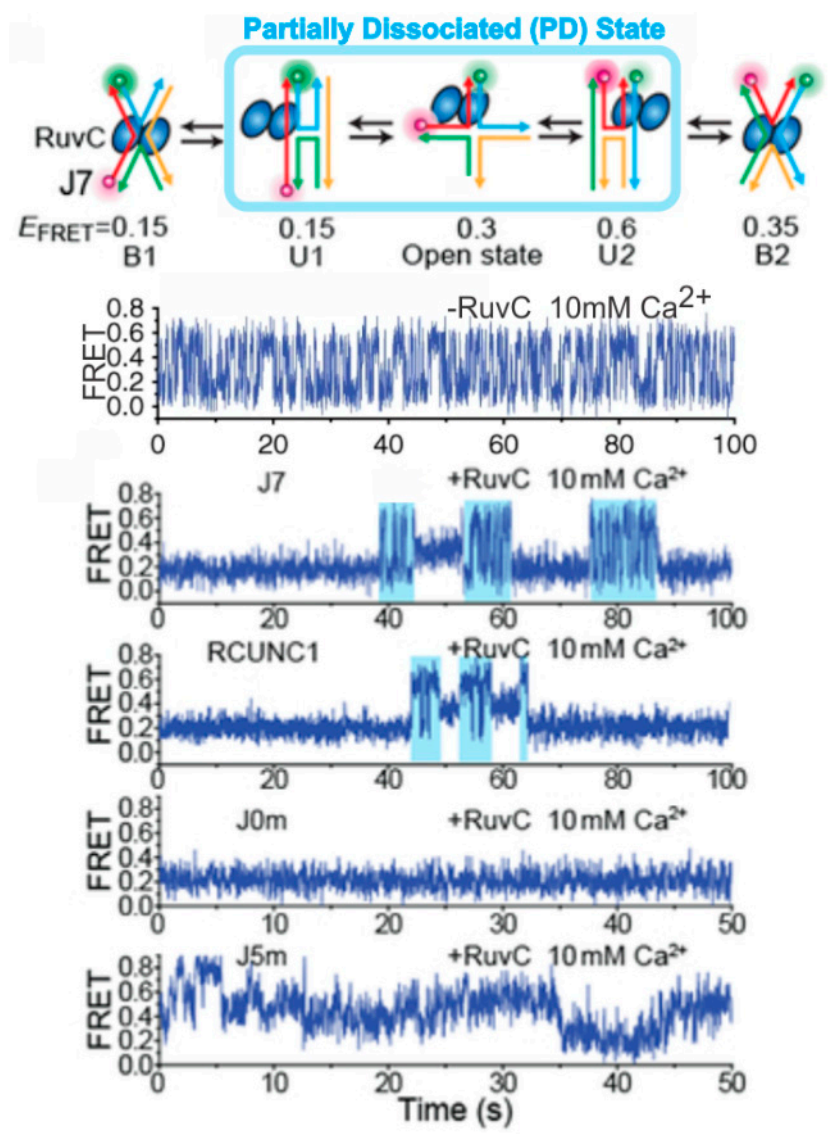

Figure 6. Single-molecule probing of RuvC-HJ interaction. The HJ resolvase RuvC binds to the HJ in such a manner that partial dissociation can occur, where one of the two RuvC dimers unbinds allowing the $\mathrm{HJ}$ to switch between its stacked conformations before being bound again by both dimers in one of the two stacked conformations. The single-molecule FRET traces correspond with, in descending order: no enzyme with a rapidly switching HJ; HJ J7 with RuvC, showing partially dissociated condition in cyan; HJ RCUNC1 with RuvC, showing the same partial dissociated (PD) state; J0m with RuvC showing no PD; and HJ J5m with RuvC. J7, RCUNC1, J0m, and J5m represent HJ with different core nucleotide sequences. The figure was adapted with permission from Nature Publishing Group [73], copyright Springer Nature 2019.

\section{Discussion and Future Perspectives}

In this review, we discussed recent work in the area of homologous recombination focusing on single-molecule fluorescence microscopy which has propelled our understanding of how HR proteins repair DNA breaks. More specifically, we described recent efforts to visualize how HR proteins operate on various DNA substrates-ssDNA, dsDNA, and Holliday junctions. We provided an overview of the methods used in these studies including FRET-based single-stranded DNA constructs, both single- and double-stranded DNA curtains, optical tweezers, and FRET-based analysis of Holliday junction analogs. These studies provided insights into the mechanism of protein binding, determined the kinetics of filament formation by various ssDNA-binding proteins, and revealed regulatory effects of protein-protein interactions on filament formation. More recent studies on HJ-binding proteins have revealed binding modes and effects of ionic environments on the binding interaction. These studies, along with bulk biochemical studies, have also started to decipher how these HR proteins contribute to the formation of branch migration complex and HJ-resolving machinery $[67,74-76]$. It is clear that single-molecule fluorescence microscopy has shed light onto the research that has dealt with the inner workings of homologous recombination machinery. While these studies have significantly improved our understanding of the HR process, due to the involvement of the same proteins in multiple functions 
and their complex interaction with other proteins for coordinated action, there is still much work to do for a complete understanding of the kinetics and mechanism of the $\mathrm{HJ}$ resolution process.

In summary, using fluorescence microscopy, significant progress has been made in understanding the roles individual HR proteins play in the overall HR process. Some inroads have been made in determining the exact roles the HR proteins play on various stages of HR. However, an understanding of the synergistic effect that multiple HR proteins have on the HR process has not yet been fully achieved. Again, more work needs to be done to characterize the way different HR proteins work together to execute the entire HR process. Particularly, the steps and proteins involved in the transition from the synaptic to post-synaptic phases in human HR need more attention. To address these knowledge gaps, several labs around the world are using fluorescence microscopy and other forms of single-molecule techniques to dissect the HR process in detail. Combining the bulk biochemical work along with the molecular-level mechanistic insights obtained from single molecule work, we envision that we will have a complete picture of HR in the near future. Once this complete picture is established, it will significantly enhance our understanding of the HR process in the context of DNA break repair, and, in the long-term, it will become possible to intelligently target HR proteins for therapies.

Funding: This work was supported by Virginia Commonwealth University (VCU Startup Grant for S.D.), and APC was paid in part by VCU Libraries open access publishing fund.

Conflicts of Interest: The authors declare no conflict of interest.

\section{References}

1. Li, X.; Heyer, W.-D. Homologous recombination in DNA repair and DNA damage tolerance. Cell Res. 2008, 18, 99-113. [CrossRef] [PubMed]

2. Camerini-Otero, R.D.; Hsieh, P. Homologous Recombination Proteins in Prokaryotes and Eukaryotes. Annu. Rev. Genet. 1995, 29, 509-552. [CrossRef] [PubMed]

3. Jasin, M.; Rothstein, R. Repair of Strand Breaks by Homologous Recombination. Cold Spring Harb. Perspect. Biol. 2013, 5, a012740. [CrossRef] [PubMed]

4. Ranjha, L.; Howard, S.M.; Cejka, P. Main steps in DNA double-strand break repair: An introduction to homologous recombination and related processes. Chromosoma 2018, 127, 187-214. [CrossRef] [PubMed]

5. Yeeles, J.T.P.; Poli, J.; Marians, K.J.; Pasero, P. Rescuing Stalled or Damaged Replication Forks. Cold Spring Harb. Perspect. Biol. 2013, 5, a012815. [CrossRef]

6. Carvalho, J.F.; Kanaar, R. Targeting homologous recombination-mediated DNA repair in cancer. Expert Opin. Ther. Targets 2014, 18, 427-458. [CrossRef]

7. Kaniecki, K.; Tullio, L.D.; Greene, E.C. A change of view: Homologous recombination at single-molecule resolution. Nat. Rev. Genet. 2018, 19, 191-207. [CrossRef]

8. Erie, D.A.; Weninger, K.R. Single molecule studies of DNA mismatch repair. DNA Repair (Amst.) 2014, 20, 71-81. [CrossRef]

9. Gauer, J.W.; LeBlanc, S.; Hao, P.; Qiu, R.; Case, B.C.; Sakato, M.; Hingorani, M.M.; Erie, D.A.; Weninger, K.R. Single-Molecule FRET to Measure Conformational Dynamics of DNA Mismatch Repair Proteins. Methods Enzymol. 2016, 581, 285-315.

10. Dupaigne, P.; Tavares, E.M.; Piétrement, O.; Le Cam, E. Recombinases and Related Proteins in the Context of Homologous Recombination Analyzed by Molecular Microscopy. Methods Mol. Biol. 2018, 1805, 251-270.

11. Bell, J.C.; Kowalczykowski, S.C. Mechanics and Single-Molecule Interrogation of DNA Recombination. Annu. Rev. Biochem. 2016, 85, 193-226. [CrossRef] [PubMed]

12. Roe, S.M.; Barlow, T.; Brown, T.; Oram, M.; Keeley, A.; Tsaneva, I.R.; Pearl, L.H. Crystal Structure of an Octameric RuvA-Holliday Junction Complex. Mol. Cell 1998, 2, 361-372. [CrossRef]

13. Ariyoshi, M.; Vassylyev, D.G.; Iwasaki, H.; Nakamura, H.; Shinagawa, H.; Morikawa, K. Atomic structure of the RuvC resolvase: A holliday junction-specific endonuclease from E. coli. Cell 1994, 78, 1063-1072. [CrossRef]

14. Hargreaves, D.; Rice, D.W.; Sedelnikova, S.E.; Artymiuk, P.J.; Lloyd, R.G.; Rafferty, J.B. Crystal structure of E.coli RuvA with bound DNA Holliday junction at 6 A resolution. Nat. Struct. Mol. Biol. 1998, 5, 441-446. [CrossRef] [PubMed] 
15. Bernstein, D.A.; Zittel, M.C.; Keck, J.L. High-resolution structure of the E. coli RecQ helicase catalytic core. Embo J. 2003, 22, 4910-4921. [CrossRef] [PubMed]

16. Xing, X.; Bell, C.E. Crystal structures of Escherichia coli RecA in a compressed helical filament. J. Mol. Biol. 2004, 342, 1471-1485. [CrossRef]

17. Brouwer, I.; Moschetti, T.; Candelli, A.; Garcin, E.B.; Modesti, M.; Pellegrini, L.; Wuite, G.J.; Peterman, E.J. Two distinct conformational states define the interaction of human RAD51-ATP with single-stranded DNA. EMBO J. 2018, 37. [CrossRef]

18. Osipiuk, J.; Li, H.; Bargassa, M.; Sahi, C.; Craig, E.A.; Joachimiak, A. Crystal structure of ATPase domain of Ssb1 chaperone, member of the HSP70 family from Saccharomyces cerevisiae. To be published, PDB 3GL1.

19. Churchill, M.E.; Tullius, T.D.; Kallenbach, N.R.; Seeman, N.C. A Holliday recombination intermediate is twofold symmetric. Proc. Natl. Acad. Sci. USA 1988, 85, 4653-4656. [CrossRef]

20. Duckett, D.R.; Murchie, A.I.H.; Diekmann, S.; von Kitzing, E.; Kemper, B.; Lilley, D.M.J. The structure of the Holliday junction, and its resolution. Cell 1988, 55, 79-89. [CrossRef]

21. Petrillo, M.L.; Newton, C.J.; Cunningham, R.P.; Ma, R.-I.; Kallenbach, N.R.; Seeman, N.C. The ligation and flexibility of four-arm DNA junctions. Biopolymers 1988, 27, 1337-1352. [CrossRef]

22. Mueller, J.E.; Newton, C.J.; Jensch, F.; Kemper, B.; Cunningham, R.P.; Kallenbach, N.R.; Seeman, N.C. Resolution of Holliday junction analogs by T4 endonuclease VII can be directed by substrate structure. J. Biol. Chem. 1990, 265, 13918-13924. [PubMed]

23. Stasiak, A.; Tsaneva, I.R.; West, S.C.; Benson, C.J.; Yu, X.; Egelman, E.H. The Escherichia coli RuvB branch migration protein forms double hexameric rings around DNA. Proc. Natl. Acad. Sci. USA 1994, 91, 7618-7622. [CrossRef] [PubMed]

24. Van Gool, A.J.; Hajibagheri, N.M.A.; Stasiak, A.; West, S.C. Assembly of the Escherichia coli RuvABC resolvasome directs the orientation of Holliday junction resolution. Genes Dev. 1999, 13, 1861-1870. [CrossRef] [PubMed]

25. Jachymczyk, W.J.; von Borstel, R.C.; Mowat, M.R.; Hastings, P.J. Repair of interstrand cross-links in DNA of Saccharomyces cerevisiae requires two systems for DNA repair: The RAD3 system and the RAD51 system. Mol. Gen. Genet. 1981, 182, 196-205. [CrossRef] [PubMed]

26. Liu, Y.; Masson, J.-Y.; Shah, R.; O’Regan, P.; West, S.C. RAD51C Is Required for Holliday Junction Processing in Mammalian Cells. Science 2004, 303, 243-246. [CrossRef] [PubMed]

27. Wyatt, H.D.M.; West, S.C. Holliday Junction Resolvases. Cold Spring Harb. Perspect. Biol. 2014, 6, a023192. [CrossRef]

28. Ogawa, T.; Shinohara, A.; Nabetani, A.; Ikeya, T.; Yu, X.; Egelman, E.H.; Ogawa, H. RecA-like Recombination Proteins in Eukaryotes: Functions and Structures of RAD51 Genes. Cold Spring Harb. Symp. Quant. Biol. 1993, 58, 567-576. [CrossRef]

29. Shinohara, A.; Ogawa, H.; Ogawa, T. Rad51 protein involved in repair and recombination in S. cerevisiae is a RecA-like protein. Cell 1992, 69, 457-470. [CrossRef]

30. Broussard, J.A.; Green, K.J. Research Techniques Made Simple: Methodology and Applications of Förster Resonance Energy Transfer (FRET) Microscopy. J. Investig. Dermatol. 2017, 137, e185-e191. [CrossRef]

31. Roy, R.; Hohng, S.; Ha, T. A practical guide to single-molecule FRET. Nat. Methods 2008, 5, 507-516. [CrossRef]

32. Marceau, A.H. Functions of Single-Strand DNA-Binding Proteins in DNA Replication, Recombination, and Repair. In Single-Stranded DNA Binding Proteins: Methods and Protocols; Keck, J.L., Ed.; Methods in Molecular Biology; Humana Press: Totowa, NJ, 2012; pp. 1-21. ISBN 978-1-62703-032-8.

33. Smith, G.R. How RecBCD enzyme and Chi promote DNA break repair and recombination: A molecular biologist's view. Microbiol. Mol. Biol. Rev. 2012, 76, 217-228. [CrossRef] [PubMed]

34. Croteau, D.L.; Popuri, V.; Opresko, P.L.; Bohr, V.A. Human RecQ Helicases in DNA Repair, Recombination, and Replication. Annu. Rev. Biochem. 2014, 83, 519-552. [CrossRef] [PubMed]

35. Smith, G.R. Homologous Recombination in Procaryotes. Microbiol. Rev. 1988, 52, 28.

36. Henrikus, S.S.; Henry, C.; Ghodke, H.; Wood, E.A.; Mbele, N.; Saxena, R.; Basu, U.; van Oijen, A.M.; Cox, M.M.; Robinson, A. RecFOR epistasis group: RecF and RecO have distinct localizations and functions in Escherichia coli. Nucleic Acids Res. 2019, 47, 2946-2965. [CrossRef]

37. Bell, J.C.; Plank, J.L.; Dombrowski, C.C.; Kowalczykowski, S.C. Direct imaging of RecA nucleation and growth on single molecules of SSB-coated ssDNA. Nature 2012, 491, 274-278. [CrossRef] 
38. Lilley, D.M.J. Holliday junction-resolving enzymes-Structures and mechanisms. FEBS Lett. 2017, 591, 1073-1082. [CrossRef]

39. Joo, C.; McKinney, S.A.; Nakamura, M.; Rasnik, I.; Myong, S.; Ha, T. Real-Time Observation of RecA Filament Dynamics with Single Monomer Resolution. Cell 2006, 126, 515-527. [CrossRef]

40. Kim, S.H.; Ragunathan, K.; Park, J.; Joo, C.; Kim, D.; Ha, T. Cooperative Conformational Transitions Keep RecA Filament Active During ATPase Cycle. J. Am. Chem. Soc. 2014, 136, 14796-14800. [CrossRef]

41. Yang, S.-H.; Zhou, R.; Campbell, J.; Chen, J.; Ha, T.; Paull, T.T. The SOSS1 single-stranded DNA binding complex promotes DNA end resection in concert with Exo1. EMBO J. 2013, 32, 126-139. [CrossRef]

42. Ragunathan, K.; Liu, C.; Ha, T. RecA filament sliding on DNA facilitates homology search. eLife 2012, 1, e00067. [CrossRef]

43. Qi, Z.; Greene, E.C. Visualizing recombination intermediates with single-stranded DNA curtains. Methods 2016, 105, 62-74. [CrossRef] [PubMed]

44. Bell, J.C.; Liu, B.; Kowalczykowski, S.C. Imaging and energetics of single SSB-ssDNA molecules reveal intramolecular condensation and insight into RecOR function. eLife 2015, 4, e08646. [CrossRef] [PubMed]

45. Galletto, R.; Amitani, I.; Baskin, R.J.; Kowalczykowski, S.C. Direct observation of individual RecA filaments assembling on single DNA molecules. Nature 2006, 443, 875-878. [CrossRef] [PubMed]

46. Kim, S.H.; Joo, C.; Ha, T.; Kim, D. Molecular mechanism of sequence-dependent stability of RecA filament. Nucleic Acids Res. 2013, 41, 7738-7744. [CrossRef]

47. Lu, C.-H.; Yeh, H.-Y.; Su, G.-C.; Ito, K.; Kurokawa, Y.; Iwasaki, H.; Chi, P.; Li, H.-W. Swi5-Sfr1 stimulates Rad51 recombinase filament assembly by modulating Rad51 dissociation. Proc. Natl. Acad. Sci. USA 2018, 115, E10059-E10068. [CrossRef]

48. Gibb, B.; Silverstein, T.D.; Finkelstein, I.J.; Greene, E.C. Single-Stranded DNA Curtains for Real-Time Single-Molecule Visualization of Protein-Nucleic Acid Interactions. Anal. Chem. 2012, 84, 7607-7612. [CrossRef]

49. Gibb, B.; Ye, L.F.; Kwon, Y.; Niu, H.; Sung, P.; Greene, E.C. Protein dynamics during presynaptic-complex assembly on individual single-stranded DNA molecules. Nat. Struct. Mol. Biol. 2014, 21, 893-900.

50. Ma, C.J.; Gibb, B.; Kwon, Y.; Sung, P.; Greene, E.C. Protein dynamics of human RPA and RAD51 on ssDNA during assembly and disassembly of the RAD51 filament. Nucleic Acids Res. 2017, 45, 749-761. [CrossRef]

51. Myler, L.R.; Gallardo, I.F.; Zhou, Y.; Gong, F.; Yang, S.-H.; Wold, M.S.; Miller, K.M.; Paull, T.T.; Finkelstein, I.J. Single-molecule imaging reveals the mechanism of Exo1 regulation by single-stranded DNA binding proteins. Proc. Natl. Acad. Sci. USA 2016, 113, E1170-E1179. [CrossRef]

52. Kaniecki, K.; Kwon, Y.; Crickard, J.B.; Sung, P.; Greene, E.C. Yeast Srs2 Helicase Promotes Redistribution of Single-Stranded DNA-Bound RPA and Rad52 in Homologous Recombination Regulation. Cell Rep. 2017, 21, 570-577.

53. Kaniecki, K.; De Tullio, L.; Gibb, B.; Kwon, Y.; Sung, P.; Greene, E.C. Dissociation of Rad51 Presynaptic Complexes and Heteroduplex DNA Joints by Tandem Assemblies of Srs2. Cell Rep. 2017, 21, 3166-3177. [CrossRef] [PubMed]

54. Brouwer, I.; Zhang, H.; Candelli, A.; Normanno, D.; Peterman, E.J.G.; Wuite, G.J.L.; Modesti, M. Human RAD52 Captures and Holds DNA Strands, Increases DNA Flexibility, and Prevents Melting of Duplex DNA: Implications for DNA Recombination. Cell Rep. 2017, 18, 2845-2853. [CrossRef] [PubMed]

55. Crickard, J.B.; Kwon, Y.; Sung, P.; Greene, E.C. Dynamic interactions of the homologous pairing 2 (Hop2)-meiotic nuclear divisions 1 (Mnd1) protein complex with meiotic presynaptic filaments in budding yeast. J. Biol. Chem. 2019, 294, 490-501. [CrossRef] [PubMed]

56. Forget, A.L.; Kowalczykowski, S.C. Single-molecule imaging of DNA pairing by RecA reveals a three-dimensional homology search. Nature 2012, 482, 423-427. [CrossRef]

57. Bianco, P.R.; Brewer, L.R.; Corzett, M.; Balhorn, R.; Yeh, Y.; Kowalczykowski, S.C.; Baskin, R.J. Processive translocation and DNA unwinding by individual RecBCD enzyme molecules. Nature 2001, 409, 374-378. [CrossRef]

58. Singleton, M.R.; Dillingham, M.S.; Gaudier, M.; Kowalczykowski, S.C.; Wigley, D.B. Crystal structure of RecBCD enzyme reveals a machine for processing DNA breaks. Nature 2004, 432, 187-193. [CrossRef]

59. Yang, L.; Handa, N.; Liu, B.; Dillingham, M.S.; Wigley, D.B.; Kowalczykowski, S.C. Alteration of $\chi$ recognition by RecBCD reveals a regulated molecular latch and suggests a channel-bypass mechanism for biological control. Proc. Natl. Acad. Sci. USA 2012, 109, 8907-8912. [CrossRef] 
60. Liu, B.; Baskin, R.J.; Kowalczykowski, S.C. DNA unwinding heterogeneity by RecBCD results from static molecules able to equilibrate. Nature 2013, 500, 482-485. [CrossRef]

61. Prasad, T.K.; Yeykal, C.C.; Greene, E.C. Visualizing the Assembly of Human Rad51 Filaments on Double-stranded DNA. J. Mol. Biol. 2006, 363, 713-728. [CrossRef]

62. Yeykal, C.C.; Greene, E.C. Visualizing the Behavior of Human Rad51 at the Single-Molecule Level. Cell Cycle 2006, 5, 1033-1038. [CrossRef]

63. Qi, Z.; Redding, S.; Lee, J.Y.; Gibb, B.; Kwon, Y.; Niu, H.; Gaines, W.A.; Sung, P.; Greene, E.C. DNA Sequence Alignment by Microhomology Sampling during Homologous Recombination. Cell 2015, 160, 856-869. [CrossRef] [PubMed]

64. Rad, B.; Forget, A.L.; Baskin, R.J.; Kowalczykowski, S.C. Single-molecule visualization of RecQ helicase reveals DNA melting, nucleation, and assembly are required for processive DNA unwinding. Proc. Natl. Acad. Sci. USA 2015, 112, E6852-E6861. [CrossRef] [PubMed]

65. McKinney, S.A.; Déclais, A.-C.; Lilley, D.M.J.; Ha, T. Structural dynamics of individual Holliday junctions. Nat. Struct. Biol. 2003, 10, 93-97. [CrossRef] [PubMed]

66. Gibbs, D.R.; Dhakal, S. Single-Molecule Imaging Reveals Conformational Manipulation of Holliday Junction DNA by the Junction Processing Protein RuvA. Biochemistry 2018, 57, 3616-3624. [CrossRef] [PubMed]

67. Karymov, M.; Daniel, D.; Sankey, O.F.; Lyubchenko, Y.L. Holliday junction dynamics and branch migration: Single-molecule analysis. Proc. Natl. Acad. Sci. USA 2005, 102, 8186-8191. [CrossRef]

68. Joo, C.; McKinney, S.A.; Lilley, D.M.J.; Ha, T. Exploring Rare Conformational Species and Ionic Effects in DNA Holliday Junctions Using Single-molecule Spectroscopy. J. Mol. Biol. 2004, 341, 739-751. [CrossRef]

69. Hohng, S.; Zhou, R.; Nahas, M.K.; Yu, J.; Schulten, K.; Lilley, D.M.J.; Ha, T. Fluorescence-Force Spectroscopy Maps Two-Dimensional Reaction Landscape of the Holliday Junction. Science 2007, 318, 279-283. [CrossRef]

70. Lahiri, S.; Li, Y.; Hingorani, M.M.; Mukerji, I. MutS $\gamma$-Induced DNA Conformational Changes Provide Insights into Its Role in Meiotic Recombination. Biophys. J. 2018, 115, 2087-2101. [CrossRef]

71. Zhao, D.; Mukerji, I.; Etson, C. Establishing a Single-Molecule FRET System for Studying DNA-Protein Interactions. Biophys. J. 2019, 116, 505a. [CrossRef]

72. Iwasa, T.; Han, Y.-W.; Hiramatsu, R.; Yokota, H.; Nakao, K.; Yokokawa, R.; Ono, T.; Harada, Y. Synergistic effect of ATP for RuvA-RuvB-Holliday junction DNA complex formation. Sci. Rep. 2015, 5, 18177. [CrossRef]

73. Zhou, R.; Yang, O.; Déclais, A.-C.; Jin, H.; Gwon, G.H.; Freeman, A.D.J.; Cho, Y.; Lilley, D.M.J.; Ha, T. Junction resolving enzymes use multivalency to keep the Holliday junction dynamic. Nat. Chem. Biol. 2019, 15, 269-275. [CrossRef] [PubMed]

74. Goyal, N.; Rossi, M.J.; Mazina, O.M.; Chi, Y.; Moritz, R.L.; Clurman, B.E.; Mazin, A.V. RAD54 N-terminal domain is a DNA sensor that couples ATP hydrolysis with branch migration of Holliday junctions. Nat. Commun. 2018, 9, 1-10. [CrossRef] [PubMed]

75. Panyutin, I.G.; Hsieh, P. The kinetics of spontaneous DNA branch migration. Proc. Natl. Acad. Sci. USA 1994, 91, 2021-2025. [CrossRef] [PubMed]

76. Hiom, K.; West, S.C. Branch migration during homologous recombination: Assembly of a RuvAB-holliday junction complex in vitro. Cell 1995, 80, 787-793. [CrossRef]

(C) 2019 by the authors. Licensee MDPI, Basel, Switzerland. This article is an open access article distributed under the terms and conditions of the Creative Commons Attribution (CC BY) license (http://creativecommons.org/licenses/by/4.0/). 